Rev. Acad. Colomb. Cienc. Ex. Fis. Nat. 42(Suplemento):177-183, octubre de 2018

doi: http://dx.doi.org/10.18257/raccefyn.756

\title{
Ciencias de la Tierra
}

\section{EL METODO DE CALDAS PARA MEDIR LA ELEVACION DE LAS MONTAÑAS}

\author{
por
}

\author{
Jorge Arias de Greiff *
}

\section{Resumen}

Arias de Greiff, J. : El método de Caldas para medir la elavación de las montañas. Rev. Acad. Colomb. Cienc. 20 (76) : 63-69, 1996. ISSN 0370-3908.

Se presenta el método de Caldas para medir la elevación de los lugares por medio de la temperatura del agua hirviendo, como lo que es: un método, no usado antes, asi se conociesen en Europa fórmulas, entre otras cosas, por las características especiales del régimen de presión atmosférica en esas latitudes y continentes.

En las condiciones particulares de la América tropical si fue posible formular el asunto como método. Se exponen otros logros posteriores, resultado del conocimiento que de esas condiciones particulares se tiene por estas tierras.

Palabras claves: Caldas - hipsometría.

\begin{abstract}
The Caldas method to measure altitude through boiling water temperature is presented. It is a method which had not been used before, even if certain formulae were already known in Europe because, among other things, the conditions of atmospheric pressure are different in those latitudes.

In the particular conditions of tropical America it was possible to propose this as a method. Some additional successes resulting from our knowledge of these particular conditions are also discussed.
\end{abstract}

Key words: Caldas - hipsometry.

Sobre el método para medir la altura de las montañas ideado por Francisco José de Caldas y Tenorio es mucho lo que se ha escrito. Sin embargo aún pueden ser útiles algunos comentarios y aclaraciones adicionales.

Observatorio Astronómico Nacional A.A. 2584 Bogotá D.C.
Es conocido el relato de la rotura de un termómetro en una excursión a las cercanías de Popayán ${ }^{1}$ y de cómo, considerando posible rehabilitarlo, decidió fabricarle una nueva escala de referencia elaborada a partir de los dos valores definidos, uno por la temperatura del hielo fundente y el otro por la temperatura de ebullición del agua. Al tratar 
de hacer esto se dio cuenta que la temperatura de ebullición dependía de la presión atmosférica. Hizo el raciocinio siguiente: si la temperatura depende de esa presión y la altura de la columna es la medida de ella, entonces se puede prescindir del barómetro y, encontrada una relación entre la temperatura de ebullición y la altura de la columna barómetrica, inferir el último dato del primero y aplicarlo a alguna de las fórmulas de nivelación barométrica que permiten encontrar la elevación de un lugar en función de la altura de esa columna. Para no perder de vista la claridad sobre el asunto que tenía don Francisco José, conviene hacer unas distinciones: Nivelación barométrica: es la determinación de la elevación de los lugares con respecto al nivel del mar o a otro lugar de comparación, usando las lecturas de la altura barométrica, debidamente corregidas de otras causas de variación como la temperatura, y aplicando estas alturas de la columna en una fórmula que las relacione con la elevación del lugar.

Nivelación termométrica : es la determinación de la elevación de un lugar por la temperatura ambiente del aire en el sitio, o también, la determinación de la elevación de un lugar por la temperatura de ebullición del agua en el sitio. Con una variante: una relación entre esa temperatura y la correspondiente altura de la columna barométrica permite continuar como nivelación barométrica.

Se le ocurrió a Caldas entonces que obviando así el uso del barómetro y reemplazándolo por la medida de la temperatura de ebullición del agua, se podría establecer un nuevo método para la determinación de la elevación de las montañas. Buscó en sus libros y nos dice Caldas que no encontró nada en ellos. No nos dijo qué era lo que buscaba: un método, la descripción detallada de los procedimientos, las normas establecidas, los consejos y recomendaciones para hallar esa elevación, o si buscaba simplemente fórmulas. Como sí encontró una fórmula, pensemos en que lo que buscaba, y no encontró, era un método. La fórmula, por lo demás, no le gustó: a ella se refirió desacreditándola. ${ }^{2}$

$1 \quad$ En el primer párrafo del "Ensayo de una Memoria sobre un nuevo método de medir la altura de las montañas", habla de esa excursión; en la nota No.3 a la publicación que del "Ensayo de una Memoria" le hizo el historiador Eduardo Posada, (Caldas, 1912), se indica que lo acompañaron Antonio Arboleda y Juan José Hurtado. En carta a Santiago Arroyo, del 5 de mayo de 1801, Caldas le indica que necesita "revolver mamotretos" en lo que se refiere al término superior de la escala termométrica, de donde se deduce que la excursión había tenido lugar poco antes.

Refiriéndose a Sigaud de la Fond dice Caldas: " $i$ Quién al ver en Sigaud (sic) de la Fond un método incierto propuesto, y deseando este físico se perfeccionen y reiteren las experiencias de este género, no había de creer que había hecho un descubrimiento, atendiendo a la época en que escribe"?. (Caldas 1978, carta No. 44).

Esa famosa frase: "no está en mis libros"3 ha sido muy usada por la beatería caldasiana para indicar la pobre situación en que se encontraba ese americano, sin darse cuenta con ello que no encontrar las cosas en los libros es una oportunidad para usar la cabeza, que no encontrar nada en los libros puede significar también que nada sabían de eso en Europa, o que si algo sabían, por razones locales de ese continente y esas latitudes, el asunto los tenía confundidos y los nativos de esas regiones, no habían podido sacar nada en claro. Por fortuna Caldas sólo encontró una fórmula en un libro, de lo contrario se hubiera enredado en el caos europeo de escalas termométricas, termómetros que se cierran cuando algún barómetro alcanza 27, 28, 29 6́ 30 pulgadas en su columna de mercurio, pulgadas unas veces inglesas y otras francesas, a lo que se agrega una sarta de fórmulas que nunca sirvieron para nada: sus autores mismos dudaban de su validez, por lo tanto inútiles para establecer y normatizar un método. Suerte del americano en no haberse topado con ese caos. Tal caos se manifiesta en Albis y Martínez (1994).

Realizó Caldas otra excursión a la hacienda de la familia en Paispamba ${ }^{4}$ e hizo observaciones de la altura de la columna en el barómetro y de la temperatura de ebullición de agua en varios lugares de los alrededores y en cerros elevados del vecindario; con esos datos y sus ideas claras sobre relación entre esas dos cantidades encontró un coeficiente que le permitía deducir la altura del barómetro del dato de temperatura, ello tomando como punto de comparación las medidas hechas en Popayán. Algo de esto había comunicado a su amigo Santiago Arroyo, radicado en Santafé; le instruyó en la toma de los datos, en verificar con granizo el estado del termómetro , ya que Santafé no tiene hielo y Popayán sí. Pronto, cuando se supo la visita del barón de Humboldt a Santafé, le advirtió no dejar traslucir nada de sus ideas al prusiano pero sí que averiguara todo lo que el viajero supiera sobre el asunto ${ }^{6}$. Algo raro debió notar el barón: que en un lugar con tantos barómetros y termómetros, le preguntaran por medidas de temperatura de agua hirviente. De algo sospechó, pues en los manuscritos de

$3 \quad$ En Obras de Caldas (Caldas, 1978)

4 En carta a Santiago Arroyo, del 5 de agosto de 1801 (Caldas, 1978, Carta No. 40), le da cuenta Caldas del éxito de esa excursión, realizada en los últimos días del mes de julio.

5 Ver las cartas $34,35,36$ y 38 (Caldas, 1978).

$6 \quad$ También en carta del 5 de julio (Caldas, 1978, Carta No. 38) le previene que se maneje con cautela y reserva. Teme que Miguel de Isla los penetre y descubra. 
su diario de viaje de los días de permanencia en Santafé hay una nota titulada "Eau bouillante" en la que plasma sus dudas sobre el asunto: que si la vasija debe ser de hierro o de barro, que si el cuello ancho o angosto, etc, con lo que demuestra que sobre el asunto las cosas no están claras, y no saber mayor cosa sobre ellas y no haberse preocupado por el temor. Por otro lado, el barón había tomado temperatura de ebullición del agua en un lugar previo del recorrido: Cumaná. En Santafé realizó esa experiencia varias veces, inclusive en Monserrate y Guadalupe. De ese lugar en adelante practica en varias localidades esa experiencia a lo largo del viaje: Contreras, Pueblo de Puracé, etc.. Algo sospechoso notó en Santafé.

Los amigos de Caldas en Popayán nada podían dejar traslucir de ese secreto, pues allí Caldas no había dicho mucho sobre su reciente descubrimiento, de modo que tampoco el barón se enteró allí de nada al respecto. Cuando llegó a Quito y en las primeras conversaciones Caldas le trató sobre la materia en forma abierta ${ }^{8}$, el barón fue sorprendido, y colocado fuera de base. El aturdido y molesto señor le dijo: que "Saussure imaginó medir las montañas por medio del termómetro sumergido en el agua hirviendo ¡bello principio! Hizo muchos experimentos sobre las montañas de Suiza y halló que 640 pies correspondian a un grado de Reaumur, siendo así que la ley de las densidades del aire es en progresión logarítmica - geométrica". Le dijo además que "se ha abandonado del todo el método de este físico por su inexactitud". Esta declaración equivocada sólo deja dos alternativas: o bien el Freiherr von Humboldt estaba desinformado, o estaba desinformando. Digamos por el momento que se trataba de lo primero, pero por el tono de autoridad, esas palabras fueron para Caldas una desinformación, que lo desilusionó. Menos mal que Humboldt se arrepintió de haber hecho eso con Caldas: ve luego sus papeles y le manifiesta: "Saussure no ha pensado como usted en agua hirviendo; sus trabajos se han limitado al temple de la atmósfera; asigna 640 pies de altura por un grado en el termómetro, y yo he observado en el Pico de Teide que da muy bien este coeficiente cuando el día es sereno y no se obra en lugares elevados"10. Con lo que el barón no deja duda de que lo había desinformado y, de intento,

\footnotetext{
$\overline{7}$ Humboldt, A von (1982) En las páginas 51 a y 73 a se encuentran notas alusivas a esas medidas.

8 Caldas (1912) página 54.

$9 \quad$ Caldas (1912), páginas 39 y 40.

10 Caldas (1912) página 40.
}

pues lo que había realizado en las islas Canarias, y tenía que saberlo, no tenía nada que ver con el bello principio del termómetro sumergido en agua hirviendo. Ahora Caldas, atenuada la desinformación por esa aclaración se entusiasma; ve claro: lo que hacen en Europa es una cosa distinta a lo que él ha ideado. Humboldt con esa aclaración limitada, luego de buscar en sus libros, los de los europeos, pone de manifiesto que él y ellos saben casi menos que Caldas, y le da a Caldas el derecho, desde ese momento, a entrar en posesión de este su pequeño descubrimiento ${ }^{11}$ y a asumir de inmediato una posición firme respecto al personaje y al asunto: "he dado un grado de perfección al método no conseguido en Europa". Caldas no supo que en Europa no es posible conseguir el mismo grado de exactitud o perfección que se logra en la América tropical. Acomete contra el método de Sausurre y defiende con claridad y lógica el que él ha ideado ${ }^{12}$. Quien no tenía las ideas claras era el señor von Humboldt: "creyó que mi coeficiente tenía los mismos defectos que el de Saussurre: pero meditando el caso, convino conmigo en esta propiedad de mi coeficiente, que lo distingue de todos ${ }^{13 "}$. Al menos de los que conocían Caldas y Humboldt. Con la lección, Caldas asume la posición de "centro" y el señor barón pasa a la "periferia". El "misionero de la ciencia", que difunde la luz de la ciencia es Caldas, así sea la luz de una ciencia débil. Ahora comienza Humboldt a entender . A entender y a alejarse de ese americano que opacaba su fulgurante presencia de americanista para quien el americano es "objeto" de estudio pero no "sujeto" que le da lecciones. Ya se desquitará haciendo de esa fecha en adelante mínima referencia a él en sus diarios. Es cierto que Humboldt incluyó en sus obras el cuadro de temperaturas, lecturas barométricas y alturas de la columna calculadas por Caldas, que habían resultado del paseo a Paispamba, pero sin una indicación explícita de que "valores calculados por el señor Caldas con el coeficiente 0.974 " fueron el resultado de un trabajo de Caldas, ni de cuál fundamento físico lo respalda, ni de para qué lo obtuvo, como si fuera cosa corriente su uso: deseo de ocultar lo que no le conviene es lo que allí se nota.

$Y$ en cuanto a Caldas, esa confrontación abierta con Humboldt lo entusiasmó, le dio confianza en sus ideas, respaldadas por sus experiencias: "Yo había suscrito con el mayor gusto a una autoridad tan respetable. Si hubiera

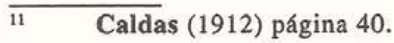

12 Caldas (1912) páginas 40 y 51.

13 Caldas (1912) página 41.
} 
autoridad contra la experiencia ${ }^{14}$," escribe Caldas motivado por la duda de Humboldt a creer en una temperatura fija de ebullición cuando la presión es la misma. Bien por el americano seguro de sí mismo y que no se deja confundir por el viajero. En su entusiasmo redacta una memoria titulada "Ensayo de una memoria titulada sobre un nuevo método para medir la altura de las montañas por medio del termómetro y el agua. hirviendo ${ }^{15}$ " de la que saca en limpio copias que envía a Antonio Arboleda, a José Ignacio de Pombo y a Mutis. En cuanto a la lección que le da a Humboldt, Caldas era la persona que en ese momento podía en el mundo darla. El era quien estaba en la frontera de ese conocimiento pues él había trabajado, el primero, esa realidad concreta de la América tropical, que permite esa perfección, así eso lo ignoraran en Europa y así Caldas ignorara que allá no tenían por qué saberlo. Esa frontera era débil y la incursión de Caldas apenas una escaramusa de validez local. A Humboldt por su parte se atribuye haber ya en esos días traspasado en América la frontera del conocimiento en otro sector de ese frente de batalla; haber descubierto las mareas atmosféricas, durante su permenencia en los llanos de Venezuela, de lo que naturalmente el sibilino barón no contó nada a Caldas. Sí le dijo de "la influencia de la luna sobre el barómetro, descubrimiento debido a Mutis"16, pero como Humboldt, descalificó el trabajo barométrico de Mutis en Santafé al anotar en su diario, -que Caldas leyó, pues en forma subrepticia y clandestina se lo mostraba Bonpland, su compañero de habitación-, que "el barómetro de Mutis es un termómetro de Ammotons" ${ }^{17}$, el asunto parece otro truco del barón. Y aquí aparece una particularidad del señor von Humboldt: Elogia sin límites a Mutis como botánico, en especial antes de la visita a Santafé; el desacrédito y burla vendrán cuando ya se encuentre lejos de Santafé. Lo elogia en algo en que Humboldt no tiene mucho que decir: botánica; para eso trajo un botánico a América, Bonpland, al que por demás olvida en su diario donde sólo lo menciona para poner de manifiesto cómo le estorban las demoras por debilidad o enfermedad del francés. A Mutis lo desacredita en algo en lo que el barón aspira a tener renombre: haber descubierto las mareas atmosféricas; debe entonces deshacerse de rivales, Mutis y Caldas, cuando hay de por medio opción creativa. Como Humboldt rá al lado de Bonpland en los descubrimientos

\footnotetext{
$14 \quad$ Caldas (1912) página 41.

15 A lo que ya se ha hecho referencia en las notas previas.

16 Caldas (1912) página 56.

17 Caldas (1978) Carta No. 60.
}

botánicos, e irá de primero (Humboldt, Bonpland, Kunth), le conviene opacar al segundo.

De Caldas hizo elogios en la Astronomía, campo en el que ninguno de los dos estaba haciendo nada creativo: ambos eran dos hábiles practicantes de métodos corrientes para determinar coordenadas geográficas de lugares, usando las posiciones de los astros; nada de rivalidad creativa y por consiguiente es posible el elogio, pero cuando lo encontró como rival creativo, en la física atmosférica y en la nivelación de las plantas, silencio, desinformación, referencia vaga y, en su momento, desinformación engañosa. Claro que mucho de esto está consignado en textos de Humboldt que no fueron revisados para ser publicados, pero por lo mismo muy dicientes en lo referente a su personalidad. Cuando Caldas, que sabía por carta recibida en un mismo correo que Mutis autorizaba el que lo acompañara en el resto de viaje, le preguntó si había recibido tal noticia, el barón le dijo: " no me dice nada el señor Mutis, no me ha escrito sobre el viaje de usted", otra vez desilusión de Caldas y otro arrepentimiento del prusiano por la mentira:"mi amigo, yo he mentido a usted: el señor Mutis me habla a la larga del asunto, pero yo, que he resuelto viejar solo, no quería dar a usted esa pesadumbre" ${ }^{18}$ su determinación era no llevarlo como compañero. Cero y van dos. Enterado Caldas de la influencia de la Luna en el barómetro, y para ese importante descubrimiento, resolvió iniciar un diario con medidas horarias de la presión atmosférica. Las que un día 1o de julio de 1802 hizo en Quito, las dejó consignadas en una carta $^{19}$. Vio entonces que para perfeccionar su método, debía realizar aún mucho trabajo $y$, lo que no debe dejar de tenerse en cuenta, emplear en ello mucho tiempo, tratándose de un sólo observador en todo un virreinato, además ocupado en recogerle quinas al señor Mutis, sin opción de hacer registros continuos durante al menos un año en lugares diversos y conocer así el régimen de mareas atmosféricas en todo su territorio. Debía realizar observaciones a más bajas y a mayores elevaciones, cosa que tenía muy en claro: " $\mathrm{Q} Q u e ́$ grado de perfección adquirirá esta teoría con observaciones sobre el Chimborazo!... Para darle la última mano es preciso ir al nivel del mar... Guayaquil, al pie, se puede decir, del inmenso Chimborazo" 20 . Pero entonces la relación ya no será lineal, o encontrará otra, esa sí, casi lineal, pero no ya entre temperatura de ebullición y altura

\footnotetext{
$18 \quad$ Caldas (1978) Carta No. 63.

19 Caldas (1978) Carta No. 69.

$20 \quad$ Caldas (1912) página 54 y 55.
} 
de la columna barométrica. Sus proyectos, motivaron que recomendara a Mutis suspendiera la remisión de la "memoria" a Cavanilles para hacerla pública ${ }^{21}$. Publicación que Caldas esperaba se hicisese antes del regreso de Humboldt a Europa. Había también cometido un error de observación: en Tagualó y Quimsacruz, sopló para quitar los vapores de agua hirviendo que rodeaban el termómetro. Los resultados de esas medidas le dieron diferencias entre las alturas barométricas leídas y las calculadas por la fórmula por lo que Caldas se desilusionó del asunto. Más tarde retomó el interés y realizó después varias operaciones de nivelación barométrica, una con observación simultánea de temperatura ambiente en Cartagena, y referida al nivel del $\operatorname{mar}^{22}$. En su informe al virrey prometió continuar en el asunto de su método ${ }^{23}$. En 1818, en forma póstuma, su memoria fue publicada en Europa, en Burdeos ${ }^{24}$. El interés en ese continente creció y muchas de las condiciones para una determinación más exacta de la temperatura de ebullición materializaron en el diseño de un aparato por Regnault, que él denominó hipsómetro, y también en los avances teóricos de la termodinámica. Así mismo se definieron las condiciones de observación: el agua debe ser químicamente pura. El vapor no debe tener vestigios de aire, así que no soplar para retirar esos vapores. En esas condiciones la tensión del vapor depende únicamente de la temperatura ${ }^{25}$. En la América tropical, donde la nivelación barométrica e hipsometría tienen opción, se realizaron trabajos para establecer el régimen de presión barométrica y preparar tablas que permitían compensar las fluctuaciones debidas a las mareas atmosféricas. Trabajos notables en este sentido fueron realizados en Colombia por Boussingault, Tomás Herrán y Juan de Dios Carrasquilla; en Venezuela, por Hann, Ugueto y por el Dr. Eduardo Rohl, en el Observatorio de Cagigal de Caracas. Esos trabajos dieron por resultado la publicación por Eduardo Rohl de tablas de corrección a la lectura barométrica para cada hora del día y la noche y para cada uno de los meses del año ${ }^{26}$. En Colombia el ingeniero y

\footnotetext{
$\overline{21} \quad$ Había pensado que su "Memoria" se publique por separado o en los "Anales de Literatura" del Abate Cavanilles. (Caldas, 1912) página 51.

22 (Caldas 1912) páginas 384 a 386

23 Publicada por la imprenta de Lavalle Joven y Sobrino, Burdeos, 1919

24 Caldas (1978) Carta No. 134

25 Rohl, E. (1941), Nuevas Tablas Barométricas, Caracas. Allí se citan los trabajos de John y los de Ugueto.

26 Rozo, Darío y Aparicio, Tomás (1944): Tablas y Fórmulas para uso del Hipsómetro y para la Refracción Atmosférica. Bogotá 1944.
}

físico Darío Rozo Martínez publicó unas tablas para el uso del barómetro y el hipsómetro, basados en una buena parte en el trabajo previo de Rohl. Por esos años también se realizaron en Puerto Rico observaciones que dieron lugar a tablas de corrección como las que se encuentran por ejemplo en el manual de Meteorología editado por Berry, Bollay y Beers en 1945; ellas registran la corrección con intervalos de 4 horas para cada una de las 4 "estaciones" del año, a las que están acostumbrados los autores de esos textos ${ }^{27}$. Muy recientemente, Jesús Antonio Eslava, ha publicado un trabajo denominado "Régimen en la presión barométrica en Colombia", muy útil para entender la viabilidad del método de Caldas (Eslava, 1995).

¿Qué es lo que les pasa entonces a los europeos?. Lo que les ocurre es que en las zonas templadas y en especial en la del hemisferio norte con sus grandes masas continentales y menores espacios oceánicos, los fenómenos ciclónicos atmosféricos cobran gran importancia. En ellos pueden presentarse diferencias de la presión del orden de 30 milibares entre el centro y los límites de esos meteoros, que recorren esas regiones con velocidad de mil a dos mil kilómetros por día, de modo que en un lugar dado esas fluctuaciones pueden ocurrrir de un día para otro, en forma aleatoria pero previsible si no se despega la mirada del barómetro. En esas regiones ese aparato se usa para predecir la llegada del mal tiempo asociado con esos fenómenos, cuando la presión está bajando o en caso contrario, predecir la llegada del buen tiempo. Esos trastornos borran cualquier regularidad de las mareas atmósfericas y hacen tenerlas en cuenta para la nivelación barométrica. También esas variaciones aleatorias sirvieron para confundir sabios.

Retornemos ahora a Darío Rozo Martínez. Este investigador, además de las tablas hipsométricas, presenta una fórmula y unas indicaciones que conforman un método para medir la altura de las montañas, con coeficiente constante de muy buena aproximación en un rango muy amplio de elevaciones. Resulta que a medida que se asciende, a iguales diferencias de la temperatura de ebullición van resultando diferencias de longitud de la columna barométrica cada vez menores y a diferencias constantes de presión atmosférica corresponden variaciones cada vez mayores de las diferencias de altura. En la primera relación el logaritmo de la presión es función del inverso de una temperatura; en la segunda la diferencia de presión es

$27 \quad$ Jenkins, G.R. (1945) "Diurnal Variation of the Meteorological Elemeents", en Handbook of Meteorology", Berry. F.A., Bollay, E., y Beers, N.R., editores, New York. 

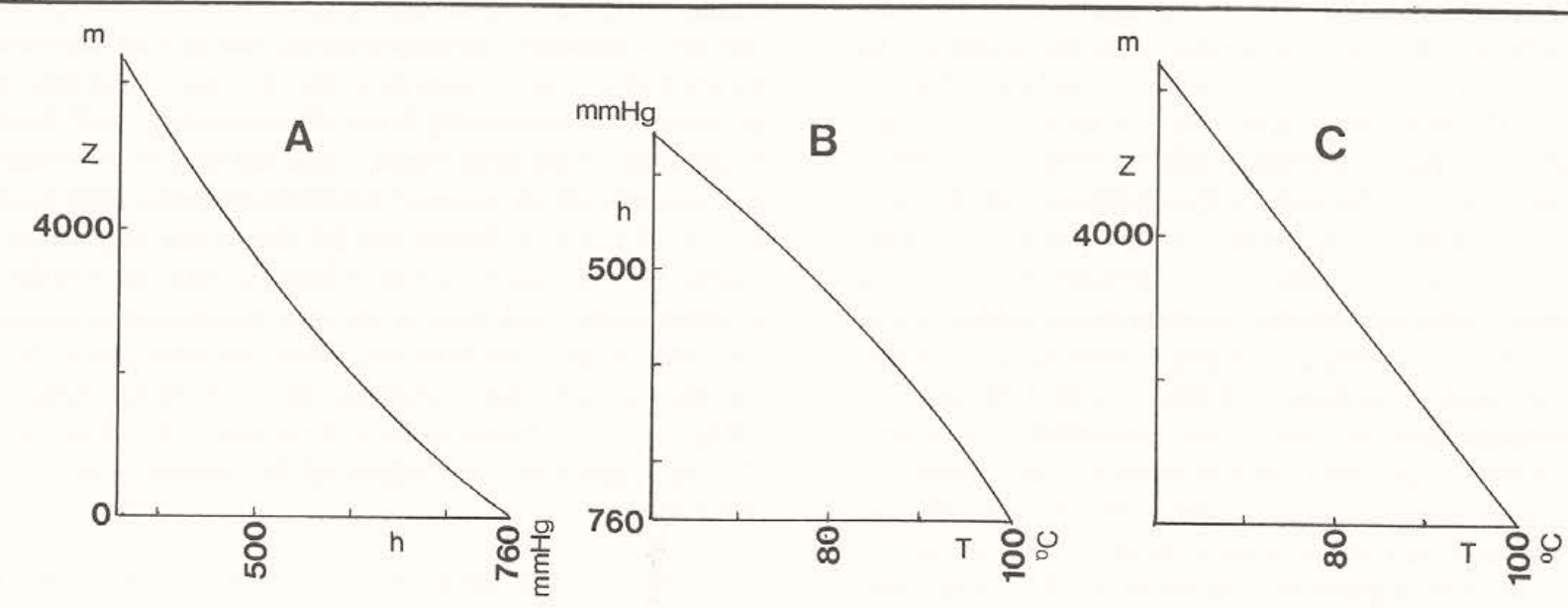

A. La relación clásica entre la presión atmosférica y la elevación de un lugar

B. La relación entre temperatura de ebullición y altura de la columna de mercurio en el barómetro.

El coeficiente hallado por Caldas que relaciona linealmente esas cantidades es una primera aproximación de alguna validez entre 1500 y 4000 metros de altura, basado en medidas tomadas en Popayán $(1760 \mathrm{~m})$ y en cerros vecinos a Paispamba (3300m). Error máximo del orden de 1,6 líneas; Caldas no se hubiera contentado con eso. El coeficiente de Caldas es la gradiente entre esa relación en la altura intermedia de $2770 \mathrm{~m}$. De todos modos Caldas necesitaba B1: Una representación de la Fórmula Antoine, con término de segundo grado que es:

$\mathrm{h}=1304.46-37.5025 \mathrm{~T}+0.320572 \mathrm{~T}^{2}$, válida sólo entre 80 y $100^{\circ} \mathrm{C}$. El error máximo es algo menor de $1 \mathrm{~mm}$ de mercurio en la columna.

C. La relación entre la temperatura de ebullición y elevación

Es una relación cuasilineal que le permite a Darío Rozo proponer la siguiente fórmula:

$\mathrm{C} 1: Z=318,3(100-\mathrm{T})+10$. Entre las 8 y las $11 \mathrm{am}$, el término 10 , se toma como 0 ; entre las 2 y las $6 \mathrm{pm}$ se toma como 20 . El error máximo entre 0 y 6400 $\mathrm{m}$ es de $3,2 \mathrm{~m}$.

C2: Una representación basada en las tablas de Darío Rozo, válida para T entre $80^{\circ}$ y $100^{\circ}$, con error mínimo de un metro, pero inválida fuera del rango, es:

$\mathrm{Z}=32106.87-324.1934 \mathrm{~T}+0,0324 \mathrm{~T}^{2}$. La manera atmosférica debe corregirse en la temperatura de ebullición (Tabla Y, Rozo \& Aparicio, 1944).

función del logaritmo de las diferencias de elevación; la relación directa entre temperatura de ebullición y elevación de montañas evita los logaritmos y puede entonces representarse muy bien por una aproximación lineal. Pero es a esta relación a la que el señor Barón asigna dos coeficientes, uno para alturas de 0 a 1.000 metros y otro para alturas hasta 7.000 metros. Sus valores son 357 metros de elevación por grado centígrado para el primer caso y 304 para el segundo, números sin ningún valor. El que sirve es el de Darío Rozo 318.3. El método con coeficiente constante no era el de Caldas: es el de Darío Rozo, con una corrección de la marea atmosférica aplicada a la elevación obtenida. En sus tablas hipsométricas, donde el coeficiente no es constante, pero casi lo es, el doctor Rozo aplica la marea atmosférica como corrección que afecta la lectura del termómetro.

Espero que estas notas le permitan a Caldas sacudirse algo de los escombros que le cayeron encima durante el bombardeo de historia internista de la ciencia europea, que le hicieron Albis y Martínez (1994).

EPILOGO - Ya pasó de moda el uso de tablas, hoy se construyen aproximaciones polinomiales, válidas para un amplio rango que representan las funciones con un mínimo error máximo, distribuido a lo largo del rango de validez, ello conseguido por las propiedades de los polinomios de Chebyshev. Para contribuir a la serie de fórmulas inútiles, he preparado dos: Para Caldas, una aproximación con término de segundo grado de las tensiones del vapor de agua y las temperaturas de esos vapores, basada en la fórmula Antoine ${ }^{28}$. Para mi profesor de Astronomia y Geodesia, ingeniero y físico Darío Rozo, otra, también con término de segundo grado, que liga

Riddick, J. A. , Bunger, W.B., (1970) “Organic Solvents”, en Techniques of Chemistry, II, Weissberger, A., editor. Tercera edición, New York. 
Rev. Acad. Colomb. Cienc. Ex. Fis. Nat. 42(Suplemento):177-183, octubre de 2018

doi: http://dx.doi.org/10.18257/raccefyn.756

temperatura de esos vapores de ebullición y altura de los lugares. La última representa los valores de elevación de su tabla con error menor de 90 centímetros. La primera representa las alturas de la columna del barómetro con un error menor del milímetro de mercurio; en ambas el rango de alturas es el del virreinato de don Francisco José de Caldas: de Guayaquil al Chimborazo.

\section{Bibliografía}

Albis, V.S., \& R. Martínez-Chavanz. 1994. Las investigaciones Meteorológicas de Caldas, En : Francisco José de Caldas, Bogotá

Caldas y Tenorio, F. J. 1912. Obras de Caldas, recopiladas y publicadas por Eduardo Posada, Bogotá. Incluye : "Observaciones sobre la verdadera altura del Cerro de Guadalupe que domina esta ciudad, dirigidas a los editores del Correo curioso. "julio y agosto de 1801", páginas 1-11. "Ensayo de una Memoria sobre un un nuevo método de medir la altura de las montañas por medio del termómetro y el agua hirviendo, seguida de un Apéndice", abril de 1802, páginas 25-47. "Memoria sobre el origen del sistema de medir montañas y sobre el proyecto de una Expedición científica", abril de 1802, páginas 49-60. "Elevación del pavimento del salón principal del Observatorio de Santafé de Bogotá" páginas 383-386.

Caldas y Tenorio, F. J. 1978. "Cartas de Caldas", publicadas por la Academia Colombiana de Ciencias Exactas, Físicas y Naturales, Bogotá.

Eslava, J. A. 1995. Régimen de la presión atmosférica en Colombia. Academia Colombiana de Ciencias Exactas, Físicas y Naturales. Colección Jorge Alvarez Lleras No. 8.

Jenkis, G. R. 1945. Diurnal Variation of the Meteorological Elements, en : "Handbook of Meteorology", New York.

O' Connor, J.F. 1945. Displacements of Pressure Systems en : "Handbook of Meteorology", New York

Rohl, E. 1941. Nuevas Tablas Barométricas preparadas especialmente para las Determinaciones Altimétricas en Venezuela y otras regiones Tropicales, Caracas.

Rozo, D. \& T. Aparicio. 1944. Tablas y fórmulas para uso del Hipsómetro y para la refracción atmosférica. Bogotá. 